\title{
Staphylococcal resistance profiles in deep infection following primary hip and knee arthroplasty: a study using the NJR dataset
}

\author{
Richard J. Holleyman ${ }^{1,2}$ (D) David J. Deehan ${ }^{1,3} \cdot$ Lucy Walker $^{2} \cdot$ Andre Charlett $^{4} \cdot$ Julie Samuel $^{3} \cdot$ Mark D. F. Shirley $^{5}$. \\ Paul N. Baker ${ }^{1,6}$
}

Received: 4 April 2018 / Published online: 15 March 2019

(c) The Author(s) 2019

\begin{abstract}
Introduction This study aimed to (1) report the rates of resistance against a variety of antibiotics for pure Staphylococcal infections, and (2) examine the impact of ALBC use at primary surgery has on resistance patterns for patients undergoing first-time revision of primary hip and knee arthroplasty for indication of infection.

Materials and methods Data from the National Joint Registry database for England and Wales were linked to microbiology data held by Public Health England to identify a consecutive series of 258 primary hip and knee arthroplasties performed between April 2003 and January 2014 that went on to have a revision for Staphylococcal deep periprosthetic infection. Multivariate binary logistic regression was used to study predictors of microorganism resistance to a range of antimicrobials.

Results After adjusting for patient and surgical factors, multivariate analysis showed the use of gentamicin-loaded bone cement at the primary surgery was associated with a significant increase in the risk of Staphylococcal gentamicin resistance (odds ratio 8.341, 95\% CI 2.297-30.292, $p=0.001$ ) and methicillin resistance (odds ratio 3.870, 95\% CI 1.319-11.359, $p=0.014)$ at revision for infection.
\end{abstract}

Conclusions Clinicians must anticipate the possibility of antibiotic resistance to ALBC utilised at primary surgery.

Keywords Arthroplasty $\cdot$ Revision $\cdot$ Infection $\cdot$ Sensitivity $\cdot$ Resistance $\cdot$ Staphylococcus

\section{Introduction}

Deep periprosthetic infection (PJI) following primary hip and knee arthroplasty is a catastrophic complication in terms of patient outcome and cost to the health service. The

Richard J. Holleyman

r.holleyman@googlemail.com

1 Institute of Cellular Medicine, Newcastle University, Newcastle upon Tyne NE2 4HH, UK

2 Health Education North East, Newcastle upon Tyne NE15 8NY, UK

3 Newcastle upon Tyne NHS Foundation Trust, Newcastle upon Tyne NE7 7DN, UK

4 Centre for Infectious Disease Surveillance and Control, Public Health England, London NW9 5EQ, UK

5 Biological, Clinical, and Environmental Systems Modelling Group, School of Biology, Newcastle University, Newcastle upon Tyne NE1 7RU, UK

6 Department of Trauma and Orthopaedics, James Cook University Hospital, Middlebrough TS4 3BW, UK implicated microorganism(s) are important determinants of clinical outcome and previous work by our group has described the epidemiology in England and Wales by linking data held by the national joint registry (NJR) to national microbiology data $[11,12]$.

In addition to identifying the microorganisms implicated in deep periprosthetic infection, it is vital to establish their antimicrobial resistance and susceptibility patterns to guide both prophylactic and therapeutic antibiotic therapies. Such therapies include systemic antibiotics (in oral and intravenous form) and local antibiotic delivery via antibiotic-loaded bone cement (ALBC) that allows delivery of high concentrations of antibiotics to the surgical site while reducing systemic side effects. However, while the use of ALBC is widespread, there is evidence to suggest that its use may lead to resistant bacterial strains $[3,15,18]$.

Using data from the National Joint Registry for England and Wales and Public Health England, this study therefore aimed to: (1) report the rates of resistance against a variety of antibiotics for pure Staphylococcal infections, and (2) examine the impact of ALBC use on resistance patterns for 
patients undergoing first-time revision of primary hip and knee replacement for indication of infection.

\section{Patients and methods}

This retrospective study linked two national databases to study antimicrobial susceptibility and resistance patterns in first-time revision for Staphylococcal deep periprosthetic infection. The methodology used to identify cases revised for periprosthetic joint infection (PJI) from the National Joint Registry for England, Wales and Northern Ireland (NJR) and subsequent linkages to microbiological data from Public Health England (PHE) has been described previously [11-13]. From the linked cohort of 579 cases (248 hips, 331 knees) a total of 258 (45\%) infected arthroplasties (105 hips, 153 knees) were identified with a pure Staphylococcal periprosthetic infection for whom antimicrobial susceptibilities were available (see Fig. 1). 157 of the cohort were male (61\%) with a mean age of 68.3 years (range 18-98 years). The majority of patients were ASA grade 2 or below (ASA $1, n=31,12 \%$; ASA $2, n=176,68 \%$ ).

\section{Dataset preparation}

Diagnosis of periprosthetic infection was determined based upon the 'reason for revision' indication recorded by the surgeon contemporaneously at the time of surgery and subsequently uploaded to the NJR. The recording of a case as infected is therefore based upon preoperative clinical review, relevant biochemical testing and preoperative microbiological investigation, as well as any perioperative surgical findings.

\section{Antimicrobial susceptibility analysis}

The study cohort of 258 cases contained more than 3,000 antimicrobial susceptibility tests. Staphylococcal susceptibility tests against each antimicrobial were classified as 'sensitive', 'intermediate', or 'resistant.' 'Intermediate' results comprise $<0.5 \%$ of results and were therefore excluded. For some patients, there were multiple tests against the same antimicrobial (e.g.,0020due to multiple samples sent at revision surgery); in such cases, where any test result was

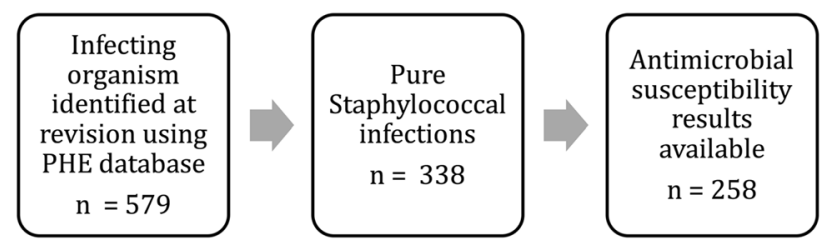

Fig. 1 Description of the study cohort 'resistant' that patient's infection was deemed resistant to the given antimicrobial (even if other samples were reported as 'sensitive').

Using the NJR data, cases were classified according to the use of bone cement. We defined any use of bone cement at primary surgery (including hybrid combinations) as cemented fixation. Cement types were further sub-classified according to their antibiotic content.

\section{Statistical analysis}

The null hypothesis was that, for a range of antimicrobials, the use of gentamicin-loaded bone cement at primary surgery would not affect Staphylococcal antimicrobial susceptibility pattern observed at time of revision. In total 31 individual antibiotics were tested against the identified samples. For the purpose of analysis we wanted to identify a group of antimicrobials for which we could analyse the influence of ABLC upon subsequent organism resistance. This was done based on the following criteria (a) antimicrobials with a minimum number of 100 antimicrobial susceptibility tests and (b) tests in which the observed proportion of resistant cases was $>10 \%$. These values were chosen to allow meaningful numbers and group sizes within our regression analysis. Preliminary analysis demonstrated that the cohort of knee arthroplasties was cemented in more than $95 \%$ of cases. The comparison group (uncemented) was therefore too small to allow robust regression analysis. As such, only hip arthroplasties were included in the regression analysis.

For each antimicrobial, a binary logistic regression model was created with resistance as the outcome and predictors being (1) the use of gentamicin-loaded bone cement vs. uncemented fixation; (2) age (grouped by $<55,55-65$, 65-75, > 75 years); (2) ASA grade (ASA 1, ASA 2, ASA 3 or 4); (3) gender; (4) body mass index (grouped by $<25$, $25-30,30-40,>40 \mathrm{~kg} / \mathrm{m} 2$ ) and (5) indication for primary surgery. Both univariate and multivariate models were performed. A $p$ value of $<0.05$ was deemed to be statistically significant.

\section{Ethical approval and data governance}

Data for this study were accessed using the standard NJR research process. The research request for this study (I.D. no. D2072003) was approved by the NJR research committee on 1 May 2014. Data linkage was achieved directly between the NJR and Public Health England. As such, no patient sensitive information (NHS no.) was held by Newcastle University as part of this analysis. After methodological review by Public Health England's confidentiality advisory group, it was determined that ethical approval was not required and the project was therefore undertaken 
as a service evaluation. The authors declare that they have no conflict of interest with respect to this study which received no funding.

\section{Results}

Susceptibility profiles for each antimicrobial tested are shown in Table 1 for the cohort of 258 arthroplasties. Not all antimicrobials were tested for in all cases. Resistance rates varied from $0 \%$ to more than $96 \%$.

Table 1 Antimicrobial resistance patterns for cohort of hip and knee arthroplasties revised for Staphylococcal infection $(n=258)$

\begin{tabular}{|c|c|c|c|}
\hline Antibiotic & No. of cases & No. resistant & $\%$ resistant \\
\hline Amikacin & 13 & 2 & 15.4 \\
\hline Amoxycillin/clavulanate & 25 & 10 & 40.0 \\
\hline Ampicillin_amoxycillin & 27 & 26 & 96.3 \\
\hline Ceftriaxone & 5 & 4 & 80.0 \\
\hline Cephalexin & 11 & 4 & 36.4 \\
\hline Chloramphenicol & 103 & 0 & 0.0 \\
\hline *Ciprofloxacin & 231 & 71 & 30.7 \\
\hline Clarithromycin & 56 & 21 & 37.5 \\
\hline *Clindamycin & 163 & 36 & 22.1 \\
\hline Co-trimoxazole & 39 & 7 & 17.9 \\
\hline Daptomycin & 51 & 3 & 5.9 \\
\hline *Erythromycin & 227 & 80 & 35.2 \\
\hline Fosfomycin & 21 & 3 & 14.3 \\
\hline *Fusidic acid & 249 & 83 & 33.3 \\
\hline *Gentamicin & 251 & 74 & 29.5 \\
\hline Imipenem & 5 & 4 & 80.0 \\
\hline Levofloxacin & 16 & 3 & 18.8 \\
\hline Linezolid & 208 & 1 & 0.5 \\
\hline *Methicillin & 253 & 100 & 39.5 \\
\hline Minocycline & 1 & 0 & 0.0 \\
\hline Moxifloxacin & 4 & 0 & 0.0 \\
\hline Mupirocin & 206 & 20 & 9.7 \\
\hline Nitrofurantoin & 29 & 1 & 3.4 \\
\hline *Penicillin & 182 & 155 & 85.2 \\
\hline Rifampicin & 239 & 23 & 9.6 \\
\hline Teicoplanin & 173 & 16 & 9.2 \\
\hline *Tetracycline & 246 & 44 & 17.9 \\
\hline Tigecycline & 66 & 0 & 0.0 \\
\hline Tobramycin & 31 & 12 & 38.7 \\
\hline *Trimethoprim & 180 & 63 & 35.0 \\
\hline Vancomycin & 208 & 3 & 1.4 \\
\hline
\end{tabular}

Asterix indicates antibiotics tested in regression analysis ( $>10 \%$ resistance, $>100$ cases)

\section{Impact of gentamicin-loaded bone cement}

Staphylococcal resistance patterns for antimicrobials fulfilling the inclusion criteria (ciprofloxacin, clindamycin, erythromycin, fusidic acid, gentamicin, methicillin, penicillin, tetracycline and trimethoprim) were compared according to the use of either gentamicin-loaded bone cement or uncemented fixation at primary hip arthroplasty. For the majority of antimicrobials tested, an increased rate of resistance was observed in association with the use of gentamicin-loaded bone cement. This was most evident for gentamicin where the use of gentamicin-loaded bone cement was associated with gentamicin resistance in 59 of 166 cases (36\%) as compared to 6 of 49 cases (12\%) where purely uncemented fixation was employed at the primary procedure (Tables 2,3 ).

For the cohort of infected hip arthroplasties, univariate binary logistic regression analysis demonstrated that the use of gentamicin-loaded bone cement at primary surgery was associated with significantly increased risk of resistance at revision surgery to erythromycin (odds ratio 2.73, 95\% CI 1.062-7.289, $p=0.037$ ), gentamicin (odds ratio 5.278, 95\% CI 1.855-15.017, $p=0.002$ ) and methicillin (odds ratio $3.252,95 \%$ CI 1.301-8.127, $p=0.012$ ). After adjusting for patient and surgical factors, multivariate analysis showed the use of gentamicin-loaded bone cement was associated with an eightfold increase in the risk of gentamicin resistance (odds ratio 8.341, 95\% CI 2.297-30.292, $p=0.001$ ) and almost four times the risk of methicillin resistance at revision for infection (odds ratio 3.870, 95\% CI 1.319-11.359, $p=0.014$ ). The use of antibiotic-loaded bone cement had no significant effect on the rates of Staphylococcal resistance to penicillin, fusidic acid, tetracycline, ciprofloxacin, erythromycin, trimethoprim or clindamycin at revision surgery when compared to uncemented fixation (Table 4).

\section{Discussion}

The principal finding of this study was that for patients undergoing revision of a primary hip arthroplasty due to Staphylococcal deep infection, there was a significantly increased rate of resistance to gentamicin and methicillin in association with use of gentamicin-loaded bone cement at primary surgery. This study did not identify any further significant patient or surgical predictors of Staphylococcal antibiotic resistance. Clinicians should be suspicious of gentamicin resistance in all cases where antibiotic-loaded bone cement is used at primary surgery and furthermore, in such cases, use of gentamicin-loaded cement may not be appropriate as a single local antibiotic therapy at revision surgery.

There is strong registry and randomised controlled trial evidence for the efficacy of ALBC in reducing the rate of both septic and aseptic revision hip and knee 

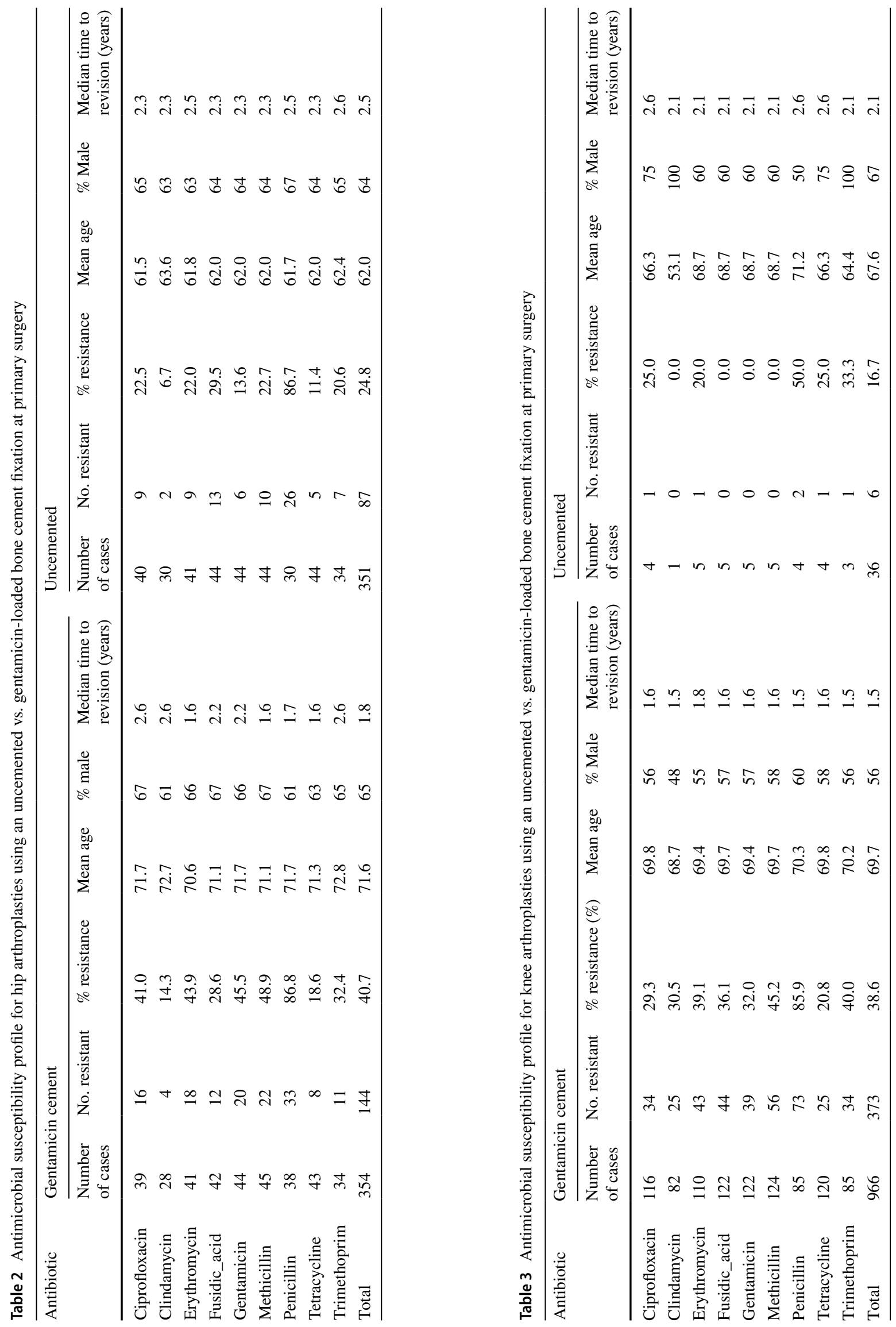
Table 4 Binary logistic regression analysis showing odds ratio for resistance to the named antibiotic given the use of gentamicin-loaded bone cement compared to uncemented fixation at primary HIP surgery

\begin{tabular}{|c|c|c|c|c|c|c|}
\hline \multirow[t]{2}{*}{ Antibiotic } & \multicolumn{3}{|l|}{ Univariate } & \multicolumn{3}{|l|}{ Multivariate } \\
\hline & Odds ratio & $95 \% \mathrm{CI}$ & $p$ value & Odds ratio & $95 \% \mathrm{CI}$ & $p$ value \\
\hline Ciprofloxacin & 2.396 & $0.900-6.376$ & 0.08 & 1.901 & $0.625-5.779$ & 0.258 \\
\hline Clindamycin & 2.333 & $0.392-13.875$ & 0.352 & 0.573 & $0.022-14.767$ & 0.737 \\
\hline Erythromycin & 2.783 & $1.062-7.289$ & $0.037 *$ & 2.329 & $0.795-6.826$ & 0.123 \\
\hline Fusidic acid & 0.954 & $0.376-2.421$ & 0.921 & 0.974 & $0.333-2.847$ & 0.961 \\
\hline Gentamicin & 5.278 & $1.855-15.017$ & $0.002 * *$ & 8.341 & $2.297-30.292$ & $0.001 * *$ \\
\hline Methicillin & 3.252 & $1.301-8.127$ & $0.012 *$ & 3.87 & $1.319-11.359$ & $0.014 *$ \\
\hline Penicillin & 1.015 & $0.247-4.166$ & 0.983 & 0.715 & $0.115-4.467$ & 0.72 \\
\hline Tetracycline & 1.783 & $0.533-5.961$ & 0.348 & 2.476 & $0.624-9.832$ & 0.198 \\
\hline Trimethoprim & 1.845 & $0.615-5.535$ & 0.275 & 2.682 & $0.729-9.872$ & 0.138 \\
\hline
\end{tabular}

$* p<0.05, * * p<0.01$ arthroplasty [5, 8]. A meta-analysis by Parvizi et al. [20] reported that the use of antibiotic-impregnated cement lowered periprosthetic infection rate by approximately $50 \%$ in primary hip arthroplasty and for revisions of previously infected hips, combinations or culture-dependent antibiotics lowered infection rates by approximately $40 \%$. Although there is an expanding body of work that demonstrates an increased rate of antibiotic resistance following the use of antibiotic-loaded bone cement, this is at odds to other studies which refute a significant relationship [7]. Hansen et al. [10] found no significant change in the epidemiology of infecting pathogens nor any notable increase in percentage resistance among organisms isolated from patients with deep infection that had received prophylactic antibiotic-loaded cement in their primary joint arthroplasty. Whether the benefit of antibiotic-loaded cement in managing and preventing deep periprosthetic infection outweighs any negative effects from subsequent resistance is unknown. However, as microorganism prevalence and resistance patterns evolve there may be a need for a greater emphasis on the choice of antibiotic used within ALBC.

Previous animal research has demonstrated an increase in gentamicin-resistant infections in rats impregnated with gentamicin-loaded cement pellets [25].

Antimicrobial resistance can occur in a variety of ways [17]. Mutational resistance can occur with various antibiotic-organism combinations. Translocation of resistance genes to mobile DNA is rarer, but can cause 'strain epidemics'. Furthermore, existing clones of resistant bacteria may simply be selected out in an antibiotic-rich environment. The process by which an antibiotic is rendered ineffective against a particular bacterial strain may also be related to the use of orthopaedic biomaterials as well as the ALBC $[1,2,9]$. ALBC has an optimum surface for colonisation, and prolonged exposure to antibiotics at sub-inhibitory levels allows mutational resistance to occur [15, 16, 19, 22, 25]. In vivo cement use (where antibiotic-loaded bone cement will remain in situ for many years), is associated with continued elution of sub-therapeutic levels of antibiotic for many years and this may further increase microbial resistance rates in these cases [4, 21, 23].

Corona et al. examined the impact of aminoglycosidecement spacers on aminoglycoside resistance in a cohort of 113 chronic periprosthetic infections following hip and knee arthroplasty due to Gram-positive cocci [6]. They reported overall gentamicin and tobramycin resistance to be 32 and $41 \%$, respectively. Additionally they found gentamicin and tobramycin resistance at re-revision to be significantly higher following the use of aminoglycoside loaded cement spacers.

In the univariate analysis, erythromycin resistance was significantly higher in patients in the ALBC group. However, after controlling for other covariates (age, gender, ASA grade, BMI, and primary surgery) the effect of ALBC as a predictor of erythromycin resistance was no longer significant. This can be attributed to the small predictive effect of ALBC of erythromycin as evidenced by the $95 \%$ confidence intervals in the univariate analysis (Table 4) becoming overwhelmed by the effects of other covariates. The methodological and data linkage limitations associated with the present study have been discussed previously [11, 12]. Unfortunately, the small numbers of cases employing uncemented fixation in the knee arthroplasty cohort precluded meaningful regression analysis of predictors of antibiotic resistance in this group. Antimicrobial susceptibility data were missing for 80 of 338 (24\%) of cases of Staphylococcal PJI. Antimicrobial susceptibility data submission is not mandatory for individual laboratories and whilst we cannot rule out selection bias, we believe the risk of this to be low as data submission to PHE is not conditional on the particular antimicrobial susceptibility profile for a given sample.

Notwithstanding the above limitations, the findings of our study are supported by existing clinical studies. Weber and Lautenbach identified gentamicin resistance in $29 \%$ of bacteria isolated preoperatively in patients revised for periprosthetic infection; this rose to $40 \%$ in cases where 
gentamicin-loaded bone cement was used. In a cohort of 91 cases, Hope et al. [14] found bacterial strains resistant to gentamicin in $88 \%$ of cases of prosthetic infection in primary hip arthroplasty where cement was loaded with this antibiotic as compared to $16 \%$ found after those where other cement types were used. An increased re-infection rate was also seen in patients presenting with a gentamicin-resistant infection. Whilst they did not study the impact of antibiotic bone cement, Stefansdottir et al. [24] performed a comprehensive analysis of 426 knee arthroplasties revised for infection using data from the Swedish arthroplasty register. Although antimicrobial susceptibility data were incomplete, the study identified a gentamicin resistance rate of approximately 34\% (20 of 58 cases of Staphylococcal infection) which compares favourably to an incidence of $29.5 \%$ ( 74 of 251 cases) observed in our study. They also reported an increase in the rate of methicillin resistance over time.

In this study we were unable to report microorganism at the species level, thus there may be cases of methicillinresistant Staphylococcus aureus (MRSA) PJI responsible for several of the observed cases of methicillin resistance. Furthermore, a significant proportion of Staphylococcal infections will be represented by Coagulase-negative staphylococci (CoNS) of which a proportion will be methicillin resistant and hence cross resistance with gentamicin and erythromycin is not unexpected; it is possible that gentamicin ALBC may lead to a selection bias for CoNS and hence the observed increase in the proportion of resistant cases may be due to an organisms' selection bias rather than development of de novo resistance within infecting organism.

In conclusion, our study reported the resistance patterns of Staphylococci strains to a broad range of antibiotics and examined the impact of gentamicin-loaded bone cement on the resultant antimicrobial susceptibility profile. Multivariate analysis showed the use of gentamicin-loaded bone cement was associated with an eightfold increase in the risk of gentamicin resistance and almost four times the risk of methicillin resistance at revision for infection. Clinicians must therefore remain vigilant to the possibility of antibiotic resistance to a given ALBC utilised at primary surgery when deciding both local and systemic antibiotic choice in the treatment of PJI at time of presentation and during revision surgery.

Acknowledgements We thank the patients and staff of all the hospitals in England, Wales and Northern Ireland who have contributed data to the National Joint Registry. We are grateful to the Healthcare Quality Improvement Partnership (HQIP), the NJR Research Subcommittee, staff at the NJR Centre and Public Health England (PHE) for facilitating this work. The authors have conformed to the NJR's standard protocol for data access and publication. The views expressed represent those of the authors and do not necessarily reflect those of the National Joint Registry Steering Committee or the Health Quality Improvement Partnership (HQIP) who do not vouch for how the information is presented.

Open Access This article is distributed under the terms of the Creative Commons Attribution 4.0 International License (http://creativeco mmons.org/licenses/by/4.0/), which permits unrestricted use, distribution, and reproduction in any medium, provided you give appropriate credit to the original author(s) and the source, provide a link to the Creative Commons license, and indicate if changes were made.

\section{References}

1. Arciola CR, Campoccia D, Montanaro L (2002 Mar) Effects on antibiotic resistance of Staphylococcus epidermidis following adhesion to polymethylmethacrylate and to silicone surfaces. Biomater 23(6):1495-1502

2. Arciola CR, Donati ME, Montanaro L (2001) Adhesion to a polymeric biomaterial affects the antibiotic resistance of Staphylococcus epidermidis. New Microbiol 24(1):63-68

3. Arizono T, Oga M, Sugioka Y (1992) Increased resistance of bacteria after adherence to polymethyl methacrylate. An in vitro study. Acta Orthop Scand 63(6):661-664

4. Bayston R, Milner RD (1982) The sustained release of antimicrobial drugs from bone cement. An appraisal of laboratory investigations and their significance. J Bone Joint Surg Br 64(4):460-464

5. Chiu F-Y, Chen C-M, Lin C-FJ, Lo W-H (2002) Cefuroximeimpregnated cement in primary total knee arthroplasty: a prospective, randomized study of three hundred and forty knees. J Bone Joint Surg Am 84A(5):759-762

6. Corona PS, Espinal L, Rodríguez-Pardo D, Pigrau C, Larrosa N, Flores X (2014) Antibiotic susceptibility in gram-positive chronic joint arthroplasty infections: increased aminoglycoside resistance rate in patients with prior aminoglycoside-impregnated cement spacer use. J Arthroplasty 29(8):1617-1621

7. Dunbar MJ (2009) Antibiotic bone cements: their use in routine primary total joint arthroplasty is justified. Orthop 32(9):660-663

8. Engesaeter LB, Espehaug B, Lie SA, Furnes O, Havelin LI (2006) Does cement increase the risk of infection in primary total hip arthroplasty? revision rates in 56,275 cemented and uncemented primary THAs followed for 0-16 years in the norwegian arthroplasty register. Acta Orthop 77(3):351-358

9. Gristina AG, Shibata Y, Giridhar G, Kreger A, Myrvik QN (1994) The glycocalyx, biofilm, microbes, and resistant infection. Semin Arthrop 5(4):160-170

10. Hansen EN, Adeli B, Kenyon R, Parvizi J (2014) Routine use of antibiotic laden bone cement for primary total knee arthroplasty: impact on infecting microbial patterns and resistance profiles. J Arthrop 29(6):1123-1127

11. Holleyman RJ, Baker P, Charlett A, Gould K, Deehan DJ (2016) Microorganisms responsible for periprosthetic knee infections in england and wales. Knee Surg Sports Traumatol Arthrosc 24(10):3080-3087

12. Holleyman RJ, Baker PN, Charlett A, Gould K, Deehan DJ (2016) Analysis of causative microorganism in 248 primary hip arthroplasties revised for infection: a study using the NJR dataset. Hip Int 26(1):82-89

13. Holleyman RJ, Deehan DJ, Charlett A, Gould K, Baker PN (2016) Does pre-operative sampling predict intra-operative cultures and antibiotic sensitivities in knee replacements revised for infection?: a study using the NJR dataset. Knee Surg Sports Traumatol Arthrosc 24(10):3056-3063 
14. Hope PG, Kristinsson KG, Norman P, Elson RA (1989) Deep infection of cemented total hip arthroplasties caused by coagulasenegative staphylococci. J Bone Joint Surg Br 71(5):851-855

15. Kendall RW, Duncan CP, Beauchamp CP (1995) Bacterial growth on antibiotic-loaded acrylic cement. A prospective in vivo retrieval study. J Arthroplasty 10(6):817-822

16. Kendall RW, Duncan CP, Smith JA, Ngui-Yen JH (1996) Persistence of bacteria on antibiotic loaded acrylic depots. a reason for caution. Clin Orthop Relat Res. 329:273-280

17. Livermore DM (2000) Epidemiology of antibiotic resistance. Intensive Care Med 26(Suppl 1):S14-S21

18. Naylor PT, Myrvik QN, Gristina A (1990) Antibiotic resistance of biomaterial-adherent coagulase-negative and coagulase-positive staphylococci. Clin Orthop Relat Res 261:126-133

19. Neut D, van de Belt H, Stokroos I, van Horn JR, van der Mei HC, Busscher HJ (2001) Biomaterial-associated infection of gentamicin-loaded PMMA beads in orthopaedic revision surgery. J Antimicrob Chemother 47(6):885-891

20. Parvizi J, Saleh KJ, Ragland PS, Pour AE, Mont MA.(2008) Efficacy of antibiotic-impregnated cement in total hip replacement. Acta Orthop 79(3):335-341

21. Picknell B, Mizen L, Sutherland R (1977) Antibacterial activity of antibiotics in acrylic bone cement. J Bone Joint Surg Br 59(3):302-307
22. Ramage G, Tunney MM, Patrick S, Gorman SP, Nixon JR (2003) Formation of Propionibacterium acnes biofilms on orthopaedic biomaterials and their susceptibility to antimicrobials. Biomaterials 24(19):3221-3227

23. Schurman DJ, Trindade C, Hirshman HP, Moser K, Kajiyama G, Stevens P (1978) Antibiotic-acrylic bone cement composites. Studies of gentamicin and Palacos. J Bone Joint Surg Am 60(7):978-984

24. Stefánsdóttir A, Johansson D, Knutson K, Lidgren L, Robertsson O (2009) Microbiology of the infected knee arthroplasty: report from the Swedish Knee Arthroplasty Register on 426 surgically revised cases. Scand J Infect Dis 41(11-12):831-840

25. Thomes B, Murray P, Bouchier-Hayes D (2002) Development of resistant strains of Staphylococcus epidermidis on gentamicinloaded bone cement in vivo. J Bone Joint Surg Br 84(5):758-760

Publisher's Note Springer Nature remains neutral with regard to jurisdictional claims in published maps and institutional affiliations. 